Auf Studienreise

\section{In Norwegen Versorgungsmodelle aus erster Hand kennen lernen}

_ In Norwegen kommen auf je 1.000 Einwohner 15,4 Menschen, die an einer Demenz leiden, in Deutschland sind es im Vergleich dazu bereits 20,3 Menschen auf 1.000 Einwohner. Doch auch in Norwegen steigt die Zahl von Menschen mit Demenz deutlich. Wie stellt sich ein hauptsächlich über Steuern finanziertes Gesundheitssystem auf diese Herausforderung ein? Welche Wege werden eingeschlagen, um unnötige Krankenhauseinweisungen von Menschen mit Demenz zu vermeiden? Welche nachhaltigen, integrierten oder koordinierten Versorgungsleistungen gibt es in Norwegen für akutstationär zu behandelnde Patienten mit Demenz?

Diese und andere Fragen stehen im Zentrum einer Studienreise, die vom 29.5. bis 2.6.2017 stattfindet. Die zweite fünftägige internationale Studienreise zur akutstationären Versorgung von Menschen mit Demenz im Krankenhaus ist Teil des Pro- gramms "Menschen mit Demenz im Akutkrankenhaus - Internationale Studienreisen", das von der Robert Bosch Stiftung gefördert wird. Es richtet sich an Personen im Gesundheitswesen, die die Versorgung von Menschen mit Demenz im Akutkrankenhaus verbessern wollen. Dazu gehören Pflegende, Ärzte, Angehörige therapeutischer Berufe, Sozialarbeiter oder Entscheider auf politischer Ebene. Vor Ort in Norwegen lernen sie innovative Modelle zur Versorgung von Menschen mit Demenz im Akutkrankenhaus aus erster Hand kennen. Das Programm „Menschen mit Demenz im Akutkrankenhaus - Internationale Studienreisen" ermöglicht seit 2015 von Experten geleitete Studienreisen zu Einrichtungen im Ausland, die mit beispielhaften Modellen die Versorgung von Menschen mit Demenz im Akutkrankenhaus verbessern konnten. Die Robert Bosch Stiftung bezuschusst die Teilnahme mit 70\% der anfallenden Kosten.

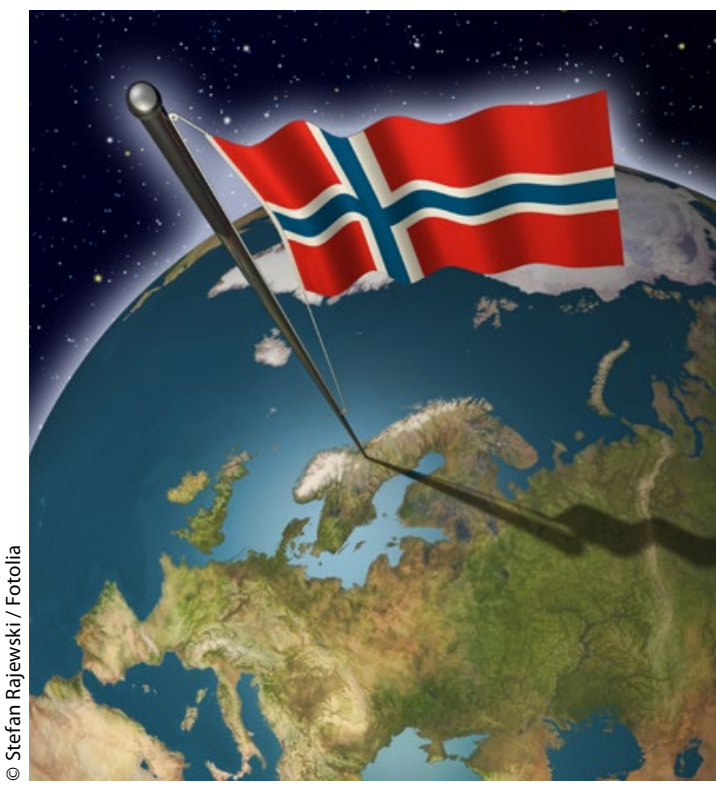

Für Mitarbeiter aus Krankenhäusern, die bereits an dem genannten Programm teilnehmen, ist die Teilnahme an der Studienreise kostenfrei.

www.g-plus.org

\section{Im Diskurs \\ Utopie Pflege 4.0}

— Tiefgreifende Veränderungen des pflegerischen Arbeitsalltags zeichnen sich ab. Ökonomisierung, Diversität und Digitalisierung in einer alternden Gesellschaft sind zentrale Begriffe. Wie kann unter diesen Bedingungen eine an den Bedürfnissen der Menschen orientierte Pflege gelingen? Welche Anforderungen an Bildungsprozesse ergeben sich daraus? Zeitgleich wandeln sich die Bedürfnisse und Voraussetzungen der Lernenden. Welche Einstellungen und Haltungen stehen bei ihnen im Vordergrund und was bedeutet dies für Lern-LehrArrangements?

Die Referierenden der 7. Fachtagung für pflegeberufliche Bildung am 31.03.2017 in Düsseldorf greifen die aktuellen Entwicklungen kritisch auf und laden zur Diskussion und zum Weiterdenken ein: _ Wie ticken Jugendliche 2016 - SINUSJugendstudie 2016
Chamäleonkompetenz. Eine habitushermeneutische Studie im Pflegemilieu

_ Werden intelligente Systeme den Pflegealltag verändern?

Die Vorträge werden durch Workshops zu aktuellen Themen der pflegeberuflichen Bildung ergänzt. In der Podiumsdiskussion wird die Frage gestellt, was aus der Ausbildungsreform der Pflegeberufe wird. Die Beiträge der Fachtagung rücken den Nutzen für die Praktiker in den Vordergrund, ohne die wissenschaftliche Fundierung dabei aus dem Blick zu verlieren.

Die Fachtagung wird von der Bundesarbeitsgemeinschaft (BAG) Pflegebildung im DBfK und dem Bildungszentrum des Universitätsklinikums Düsseldorf veranstaltet.

www.dbfk.de
Alles richtig?

Die korrekten Antworten der PflegeKollegs lauten:

Medikamentensicherheit $1 c, 2 a, 3 a, 4 b, 5 c, 6 b, 7 c, 8 b, 9 a, 10 c$

Schmerz \& Psyche 1a, 2a, 3b, 4c, 5a, 6b, 7a, 8c, 9a, 10c

Vorschau! Die PflegeKollegs im Monat April:

Diabetes

Demenz - Ernährung - Schnittstellenmanagement

Geriatrie

Schluckstörungen - Gender - Traumatologie 